\title{
Number of Diseased Coronary Artery Vessels
}

National Cancer Institute

\section{Source}

National Cancer Institute. Number of Diseased Coronary Artery Vessels. NCI Thesaurus.

Code C99996.

Number of major epicardial vessels with greater than or equal to $70 \%$ reduction in diameter or greater than or equal to $50 \%$ reduction in cross-sectional area. (ACC) 